\title{
$A b$ initio based method to study structural phase transitions in dynamically unstable crystals, with new insights on the $\beta$ to $\omega$ transformation in titanium
}

\author{
D. Korbmacher,,${ }^{1, *}$ A. Glensk, ${ }^{1}$ A. I. Duff, ${ }^{2}$ M. W. Finnis,${ }^{3}$ B. Grabowski, ${ }^{1}$ and J. Neugebauer ${ }^{1}$ \\ ${ }^{1}$ Max-Planck-Institut für Eisenforschung GmbH, Max-Planck-Strasse 1, D-40237 Düsseldorf, Germany \\ ${ }^{2}$ Scientific Computing Department, STFC Daresbury Laboratory, Hartree Centre, Warrington WA4 4AD, United Kingdom \\ ${ }^{3}$ Department of Materials and Department of Physics, Thomas Young Centre, Imperial College London, \\ Exhibition Road, London SW7 2AZ, United Kingdom
}

(Received 23 July 2018; revised manuscript received 3 December 2018; published 12 September 2019)

\begin{abstract}
We present an approach that enables an efficient and accurate study of dynamically unstable crystals over the full temperature range. The approach is based on an interatomic potential fitted to ab initio molecular dynamics energies for both the high- and low-temperature stable phases. We verify by comparison to explicit $a b$ initio simulations that such a bespoke potential, for which we use here the functional form of the embedded atom method, provides accurate transformation temperatures and atomistic features of the transformation. The accuracy of the potential makes it an ideal tool to study the important impact of finite size and finite time effects. We apply our approach to the dynamically unstable $\beta$ (bcc) titanium phase and study in detail the transformation to the low-temperature stable hexagonal $\omega$ phase. We find a large set of previously unreported linear-chain disordered (LCD) structures made up of three types of $[111]_{\beta}$ linear-chain defects that exhibit randomly disordered arrangements in the $(111)_{\beta}$ plane.
\end{abstract}

DOI: 10.1103/PhysRevB.100.104110

\section{INTRODUCTION}

Besides thermodynamically stable and metastable phases, dynamically unstable phases are important for the description of crystalline materials [1-3]. A dynamically unstable phase refers to a saddle point in the potential energy surface at $T=0 \mathrm{~K}$ as a function of atomic coordinates. If the atoms are unconstrained, the smallest deviation from their ideal lattice positions will allow the system to slide into a neighboring energy minimum [4]. The attempt to calculate frequencies of vibration within the harmonic or quasiharmonic approximation in a dynamically unstable lattice reveals the saddle point in the form of negative eigenvalues of the dynamical matrix, representing imaginary frequencies. This makes the (quasi)harmonic methods for calculating phonon frequencies and hence free energies unworkable. Methods going beyond such low-temperature approximations are required to treat dynamically unstable phases. The main aim of this paper is to introduce an $a b$ initio based method to study structural phase transitions in dynamically unstable crystals. To demonstrate our approach we choose the important example of the $\beta$ (bcc) phase and its transition to the $\omega$ phase (see Fig. 1) exhibited, e.g., by titanium or zirconium and their alloys.

One possible approach to study dynamically unstable systems is to employ molecular dynamics (MD) based on interatomic potentials, for example the embedded atom method (EAM), enabling the simulation of large system sizes and long simulation times. In this way, the high-temperature stabilization of dynamically unstable phases can be explicitly investigated. However, the accuracy of interatomic potentials,

*Corresponding author: d.korbmacher@mpie.de in particular for properties that were not considered in the fitting procedure or given only small weight, is problematic. In this respect, $a b$ initio simulations that consider explicitly electronic interactions are superior. Several approaches have been developed to overcome the difficulties associated with dynamically unstable systems and to carry out the necessary sampling of phase space, at least approximately, while capturing electronic effects at the level of density-functional theory (DFT) [5]. For example, the self-consistent ab initio lattice dynamics (SCAILD) method [6,7] or the temperature dependent effective potential (TDEP) method [8] stabilize the system by utilizing effective harmonic potentials fitted to the high-temperature stable phase. Another approach based on harmonic lattice dynamics stabilizes the dynamically unstable system by a reduction of the degrees of freedom [9]. The anharmonic potential cluster expansion [10] as well as the recently developed piecewise polynomial potential partitioning $\left(\mathrm{P}^{4}\right)$ method $[11,12]$ provide an alternative route to study dynamically unstable systems by expanding the potential energy surface in terms of a Hamiltonian composed of effective cluster interactions or piecewise polynomials fitted to DFT energies.

Progress has been also made on approaches that enable full sampling of phase space and numerical determination of the free energy at the DFT level. For example, the two-stage upsampled thermodynamic integration using Langevin dynamics (TU-TILD) [13] method uses an optimized interatomic potential (e.g., EAM) as an efficient bridge for thermodynamic integration from quasiharmonic to anharmonic free energy, followed by up-sampling to obtain DFT accuracy. The efficiency of the TU-TILD potential comes from the specific fitting procedure where only the most relevant part of the phase space enters, i.e., DFT MD energies for a single, target 

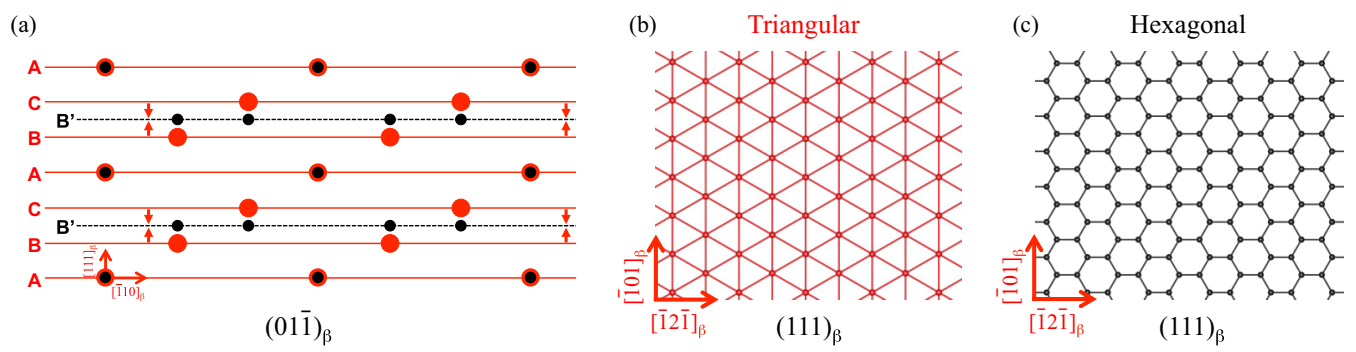

FIG. 1. Schematic representation of the conventional understanding of the $\beta$ to $\omega$ phase transformation. (a) The stacking sequence of the $\beta$ structure (red dots) is $\mathrm{ABCABC}$ and $\mathrm{AB}$ ' $\mathrm{AB}$ ' $\mathrm{AB}$ ' for the $\omega$ structure (black dots). The phase transformation occurs by two planes coalescing to one new plane, e.g., B and C to B'. There are two symmetrically equivalent variants $\mathrm{C}, \mathrm{A} \rightarrow \mathrm{C}^{\prime}$ and $\mathrm{A}, \mathrm{B} \rightarrow \mathrm{A}$ '. (b) and (c) show the pattern which arises in the $(111)_{\beta}$ projection with (b) a triangular topology for planes A, B, C and (c) a hexagonal topology for plane B' (in $\omega$ only).

phase. This fitting strategy is in contrast to the one employed for the aforementioned conventional interatomic potentials, where transferability to different phases is desired. In the two-optimized references thermodynamic integration using Langevin dynamics (TOR-TILD) [14] method, the concept of TU-TILD was extended to phase transitions, in particular to the solid to liquid transition, by introducing two optimized potentials fitted to DFT MD energies of each of the phases.

In the present paper, we show that the concept of fitting a tailored interatomic potential is well suited to describe dynamically unstable systems. We focus on the prototype $\beta$ phase in Ti and its transformation to the $\omega$ phase neglecting the small strain contribution [15]. The $\beta$ to $\omega$ transformation is known to occur in pure $\mathrm{Ti}$ at higher pressures (above $8 \mathrm{GPa}$ ) and also in $\mathrm{Ti}$ alloys (e.g., upon alloying with $\mathrm{V}$ or $\mathrm{Nb}$ ) [16-22]. The $\omega$ phase plays an important role in the design of Ti alloys where it can have detrimental [23] or beneficial [21] properties. We study here the $\beta$ to $\omega$ transformation in an experimentally unreachable regime, i.e., at zero pressure in pure Ti, by suppressing the transformation to the $\alpha$ (hcp) phase through constant volume calculations. This approach renders the involved DFT MD calculations feasible and the obtained results can be expected to be qualitatively transferable to the high pressure and concentrated regimes [24]. Applying this approach, we find-besides the three variants of the perfect $\omega$ structure (Fig. 1), which are alternative commensurate structures to the $\beta$ lattice-numerous highly defective $\omega$ structures in which the stacking sequence is broken.

To properly describe the $\beta$ to $\omega$ transformation including the defective structures, we extend the fitting strategy that has been applied in the previous TU-TILD and TOR-TILD methods. In each of these methods, a potential is fit to a single, specific phase. Here, we fit our potential over a large enough temperature range to capture the relevant energetics of both the $\beta$ and $\omega$ phases, the corresponding transformation, and the defective structures. Note that this fitting strategy still stands out of the conventional fitting strategy that aims to achieve transferability to all relevant phases and defect structures, i.e., transferability to an extensive part of phase space. In contrast, our potential is on purpose restricted to a small part of phase space spanning two, geometrically closely related phases (see Fig. 1). We show that this potential, referred to as $\beta \omega$-EAM, offers an important advantage, i.e., efficient access to large length and timescales, enabling the study of finite size effects on the transformation behavior. The obtained results demonstrate that the $\beta$ to $\omega$ transformation is accompanied by a hysteresis (consistent with the first order character [25]) that vanishes for experimental timescales. The results further suggest that the highly defective $\omega$ structures can be viewed as a new set of structures, which we refer to as linear-chain disordered (LCD) structures, that are related to perfect $\omega$ by the formation of linear-chain defects along the $[111]_{\beta}$ direction.

\section{COMPUTATIONAL DETAILS}

The $\beta \omega$-EAM potential was fitted to DFT MD simulations at different, fixed volumes (corresponding lattice constants: $3.24,3.27$, and $3.31 \AA$ ) and different temperatures $(850,1350$, 1600 , and $1941 \mathrm{~K}$ ) in a $3 \times 3 \times 3$ cubic bcc supercell with 54 atoms. The chosen temperature range covers the stability region of the $\beta$ and $\omega$ phase. In particular, at $850 \mathrm{~K}$ the defective $\omega$ structure is observed as discussed in detail in Sec. III A. A total of 1200 energies was used in the optimization and a resulting mean-square deviation of $10.6 \mathrm{meV} /$ atom was obtained. Fitting was performed using the MEAMFIT2 code $[26,27]$. An attempt was made to optimize a referencefree modified EAM (RF-MEAM) potential [26] but no improvement was found upon the EAM parametrization. The optimized parameters of the $\beta \omega$-EAM potential are given in Ref. [28].

The DFT MD simulations were performed using the Vienna $a b$ initio simulation package (VASP) $[29,30]$ and the

TABLE I. Parameters used in the DFT MD calculations. All calculations were performed using Fermi-Dirac broadening with a parameter of $0.1 \mathrm{eV}$. The first row corresponds to the parameters used for the fitting database.

\begin{tabular}{lccccl}
\hline \hline Sec. & Fig. & Supercell & Atoms & Initial structure & Sim. time (ns) \\
\hline- & - & $3 \times 3 \times 3$ & 54 & $\beta$ & 0.025 \\
III A & 2 & $3 \times 3 \times 3$ & 54 & $\beta$ & 0.025 \\
III A & 5 & $3 \times 3 \times 3$ & 54 & $\beta$, perfect $\omega$ & 0.025 \\
III B & 7 & $6 \times 6 \times 6$ & 432 & $\beta$ & 0.005 \\
III B & 8 & $2 \times 2 \times 2$ & 16 & $\beta$ & 0.05 \\
III B & 8 & $3 \times 3 \times 3$ & 54 & $\beta$ & 0.025 \\
III B & 8 & $4 \times 4 \times 4$ & 128 & $\beta$ & 0.005 \\
III B & 8 & $6 \times 6 \times 6$ & 432 & $\beta$ & 0.005 \\
\hline \hline
\end{tabular}


TABLE II. Parameters used in the $\beta \omega$-EAM MD simulations.

\begin{tabular}{lccccl}
\hline \hline Sec. & Fig. & Supercell & Atoms & Initial structure & Sim. time (ns) \\
\hline III A & 5 & $3 \times 3 \times 3$ & 54 & $\beta$ & 0.05 \\
III B & 7 & $6 \times 6 \times 6$ & 432 & $\beta$, LCD & 0.05 \\
III B & 8 & $2 \times 2 \times 2$ & 16 & $\beta$ & $0.05 / 50$ \\
III B & 8 & $3 \times 3 \times 3$ & 54 & $\beta$ & $0.025 / 50$ \\
III B & 8 & $4 \times 4 \times 4$ & 128 & $\beta$ & $0.01 / 50$ \\
III B & 8 & $6 \times 6 \times 6$ & 432 & $\beta$, LCD & $0.01 / 50$ \\
\hline \hline
\end{tabular}

projector augmented wave (PAW) method [31] as implemented in VASP. The generalized-gradient approximation with the Perdew-Burke-Ernzerhof parametrization [32] was used for the exchange-correlation functional. A PAW potential including the semi core $p$ orbitals with an electronic configuration of $3 p^{6} 3 d^{2} 4 s^{2}$ was chosen [33]. Further details are given in Table I.

The $\beta \omega$-EAM MD simulations were performed using the Large-scale atomic/molecular massively parallel simulator (LAMMPS) [34]. Further details are given in Table II.

All MD simulations were performed in an $N V T$ ensemble, i.e., at constant volume conditions. To control the temperature during the MD simulation the Langevin thermostat with a friction parameter of $0.01 \mathrm{fs}^{-1}$ was used. The time step for all MD simulations was set to $5 \mathrm{fs}$. Except for the calculations for the DFT fitting database, we used the equilibrium volume of the $\beta$ phase (lattice constant: $3.253 \AA$ ). The constant volume conditions prevent the transformation to the $\alpha$ Ti phase. This transformation requires a much larger strain deformation compared to the $\beta$ to $\omega$ transformation which requires only an atomic shuffling [15]. As we will discuss, a transformation to a constrained cubic hcp structure (excluding strain deformation) is suppressed as well.
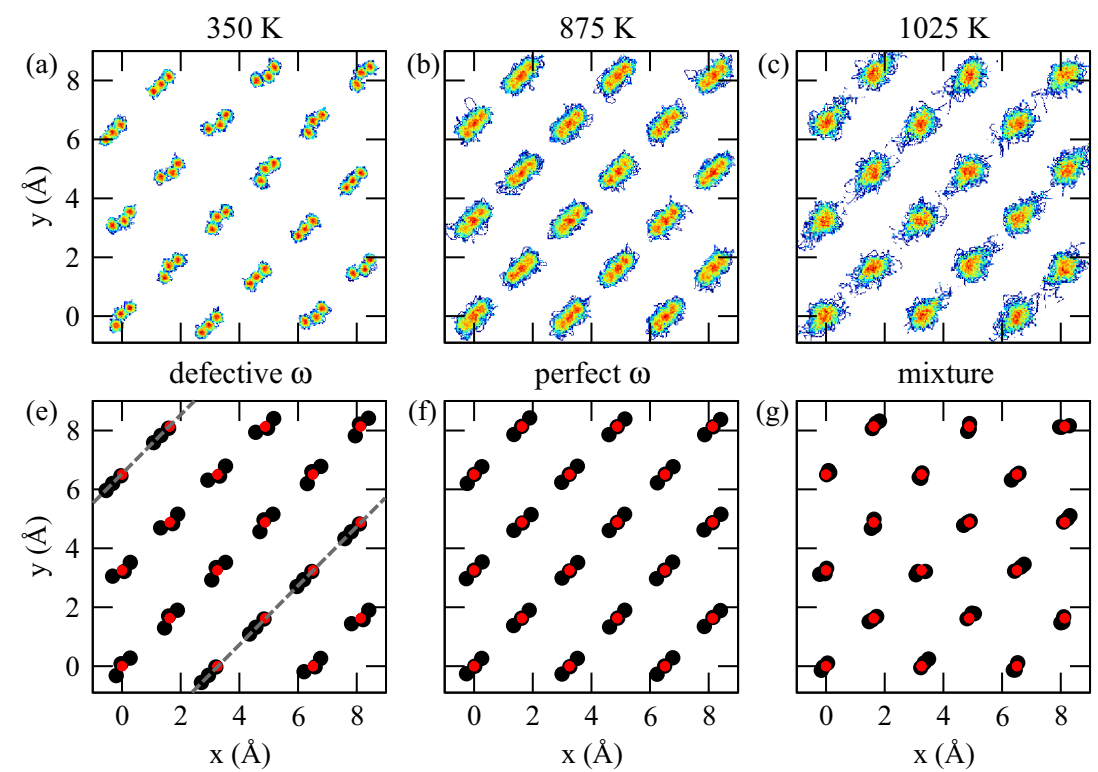

perfect $\omega$

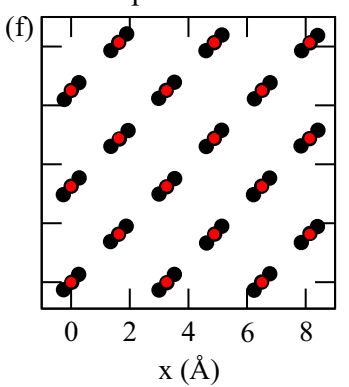

\section{RESULTS AND DISCUSSION}

\section{A. The $\beta$ to $\omega$ phase transformation from DFT}

We start with an analysis of the $\beta$ to $\omega$ transformation using "pure" DFT MD simulations in a $3 \times 3 \times 3$ cubic bcc supercell with 54 atoms. This supercell size enables DFT MD simulations in a realistic time frame. While, as we will show later, the size of this cell is too small to capture all details of the transformation its purpose is twofold: (i) to obtain a basic understanding of the transformation fully from DFT and (ii) to set a well-defined reference for fitting and testing the $\beta \omega$-EAM potential.

Figures 2(a)-2(d) show the atomic distributions of the DFT MD runs projected onto the $(001)_{\beta}$ plane at different temperatures. Averaged mean positions are shown by the black dots in Figs. 2(e)-2(h). The red dots represent the equilibrium coordinates of the $\beta$ (bcc) structure. At higher temperatures, e.g., $1941 \mathrm{~K}$, there is virtually no difference between the initial $\beta$ and the final mean positions confirming the stability of the $\beta$ phase. At lower temperatures, e.g., $875 \mathrm{~K}$, the mean positions of some atoms are shifted. Thus, whereas at high temperatures the $\beta$ structure is stabilized by anharmonicity, a secondary structure arises at low temperatures. The obtained low-temperature structure corresponds only in some cases to the perfect $\omega$ structure (Fig. 1) as known from previous works $[15,25,35]$; in most cases, when initializing with $\beta$ positions, it is a defective $\omega$ structure.

To better reveal the $\omega$ structure, the projection plane needs to be changed from $(001)_{\beta}$ to $(01 \overline{1})_{\beta}$, with the actual plane depending on the direction of the symmetry breaking. The modified perspective is shown in Fig. 3(a) for the initial and mean positions of the MD run at $875 \mathrm{~K}$. For this temperature the simulation does reproduce the transformation from the $\beta$ to the perfect $\omega$ structure. The perfect $\omega$ unit cell is highlighted

FIG. 2. Analysis of the DFT MD simulations in a $3 \times 3 \times 3$ cubic bcc supercell with 54 atoms. [(a)-(d)] 2D histograms of the atomic distributions projected onto the $(001)_{\beta}$ plane at representative temperatures $\left(T_{\exp }^{\text {melt }}=1941 \mathrm{~K}\right.$ is the experimental melting point). [(e)-(h)] Corresponding mean atomic positions (black dots) in comparison to the initial $\beta$ positions (red dots). Note that for this supercell and projection three mean positions overlap for the high-temperature $\beta$ structure in (h). Two of these mean positions are shifted and become visible in the low-temperature $\omega$ structures in (e) and (f). 

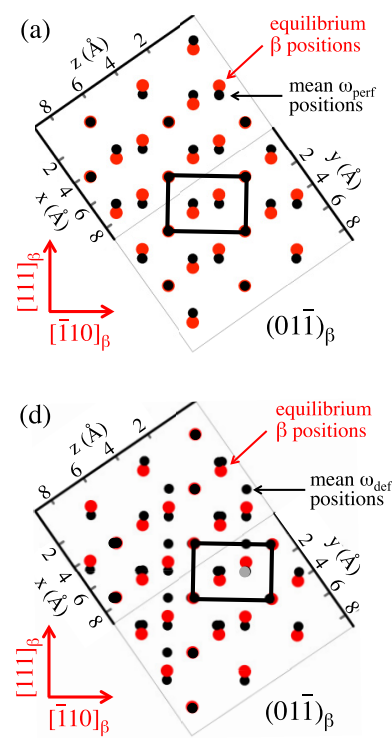
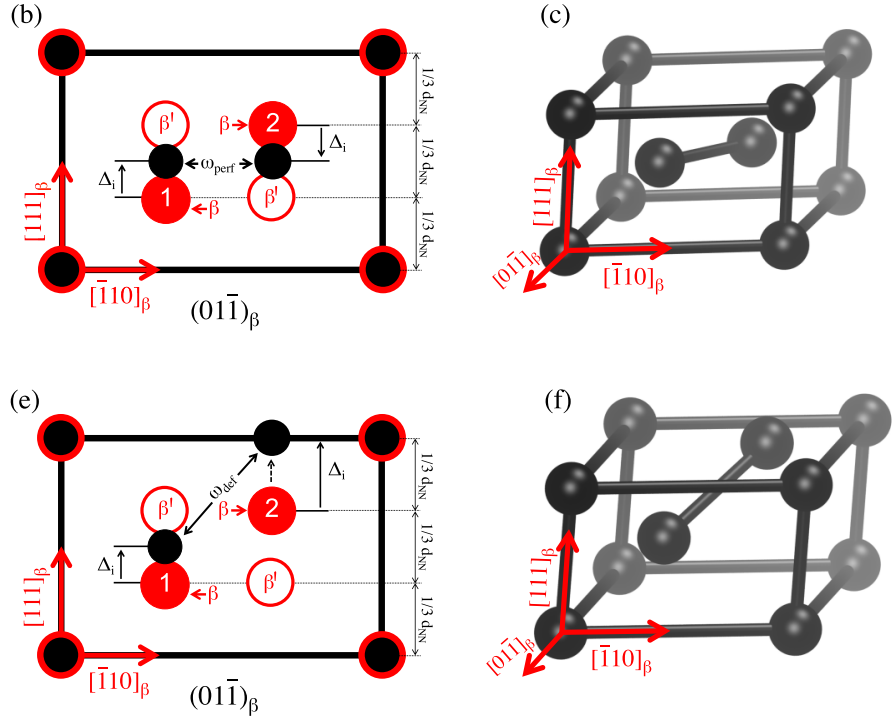

FIG. 3. Illustration of the perfect (top row; $\left.\omega_{\text {perf }}\right)$ and defective (bottom row; $\left.\omega_{\text {def }}\right) \omega$ structure. [(a) and (d)] Projection onto the $(01 \overline{1})_{\beta}$ plane of the initial $\beta$ positions (red dots) and mean positions (black dots) from the DFT MD simulations in a $3 \times 3 \times 3$ supercell with 54 atoms at $875 \mathrm{~K}$ (perfect $\omega$ ) and $350 \mathrm{~K}$ (defective $\omega$ ), respectively. The black rectangles highlight a perfect and defective $\omega$ unit cell, enlarged in (b) and (e), and illustrated three-dimensionally in (c) and (f). The gray dot in (d) indicates an atom lying in a plane behind the cell highlighted by the black rectangle; this atom is therefore not shown in (e) and (f).

by the black rectangle in Fig. 3(a), and enlarged in Figs. 3(b) and 3(c). As compared to the $\beta$ structure, one out of three atoms remains in the equilibrium position, whereas two out of three atoms perform a shift along the $[111]_{\beta}$ direction by a distance of $1 / 6 d_{\mathrm{NN}}$, with $d_{\mathrm{NN}}=2.82 \AA$ the nearest-neighbor distance in $\beta$. In the employed 54 atomic supercell, 18 atoms (one third) remain in $\beta$ positions and 36 atoms (two-thirds) perform the shift.

Most of the low-temperature MD runs starting from $\beta$ positions do not show the perfect $\omega$ structure, but instead a defective $\omega$ structure. Mean positions for such a structure are shown in Fig. 3(d) with the black rectangle highlighting an $\omega$ unit cell containing a defect. The enlarged representation of the defective $\omega$ unit cell in Figs. 3(e) and 3(f) clarifies that one of the two middle atoms (labelled " 2 ") is shifted by $1 / 3 d_{\mathrm{NN}}$ into the opposite direction as compared to the corresponding displacement for a perfect $\omega$ cell. This opposite shift occurs in all consecutive $\omega$ cells along the $[111]_{\beta}$ direction as visualized in Fig. 4(a) (gray dashed lines). Figure 4(a) makes also clear that not every $\omega$ unit cell in a defective $\omega$ structure contains a defect, only every third. Moreover, there is a regular pattern of how the defective and perfect $\omega$ unit cells are arranged within the $(111)_{\beta}$ plane as visualized in Fig. 4(b).

Based on these geometric considerations it seems appropriate to refer to the defects occurring in a defective $\omega$ structure as linear-chain defects along the $[111]_{\beta}$ direction. There are three linear-chain defects in the $3 \times 3 \times 3$ supercell. (Note that these three defects are of the same type. Larger supercells will enable the simultaneous occurrence of different types of linear-chain defects; Sec. III C.) Every linear-chain defect introduces small relaxations in the mean positions of the neighboring atoms within the $(111)_{\beta}$ plane as visualized in Fig. 2(e) (see the deviations from the diagonal arrangement of the black dots next to the linear-chain defects represented by the gray dashed lines). The regular pattern observed in the $(111)_{\beta}$ plane could be interpreted as an ordering of the defects, but we will show (Sec. IIIC) that this pattern is stabilized only by the small supercell size.

To be able to conveniently distinguish the different structures from each other as a function of temperature, we introduce a structure descriptor. One could assume that the phonon eigenvector associated in earlier works $[15,25,35]$ with the $\beta$ to $\omega$ transformation would be appropriate for this purpose. However, such a choice works well only for the perfect $\omega$ structure, but not for the defective one. It is also not practical to use the displacement vector corresponding to the defective $\omega$ structure as a descriptor, because the arrangement of the linear-chain defects changes for larger system sizes.

The most effective structure descriptor turns out to be

$$
\Delta=\frac{1}{N} \sum_{i}^{N} \Delta_{i}, \quad \Delta_{i}=\left\|\left\langle\mathbf{R}_{i}\right\rangle_{T}-\mathbf{R}_{i}^{\beta}\right\|_{[111]_{\beta}},
$$

where $N$ is the number of atoms in the supercell, $\left\langle\mathbf{R}_{i}\right\rangle_{T}$ the mean position of atom $i$ at temperature $T, \mathbf{R}_{i}^{\beta}$ the equilibrium position of atom $i$ in the $\beta$ structure, and $\|\mathbf{v}\|_{[111]_{\beta}}$ denotes the norm of the projection of a vector $\mathbf{v}$ onto the symmetry broken direction $[111]_{\beta}$. The equilibrium positions $\mathbf{R}_{i}^{\beta}$ are adjusted for each run, i.e., out of the set of symmetrically equivalent equilibrium $\beta$ positions the ones closest to the mean positions $\left\langle\mathbf{R}_{i}\right\rangle_{T}$ are chosen as $\mathbf{R}_{i}^{\beta}$. The atomic displacement $\Delta_{i}$ is illustrated in Fig. 3(b) for a perfect and in Fig. 3(e) for a defective shift. Note that $\Delta_{i}$ takes only positive values.

The temperature dependence of the structure descriptor for the DFT MD simulations in the $3 \times 3 \times 3 \mathrm{bcc}$ supercell is shown in Fig. 5. The gray squares indicate results of the simulations discussed so far, i.e., runs for which the $\beta$ positions were used as starting positions. We have performed 

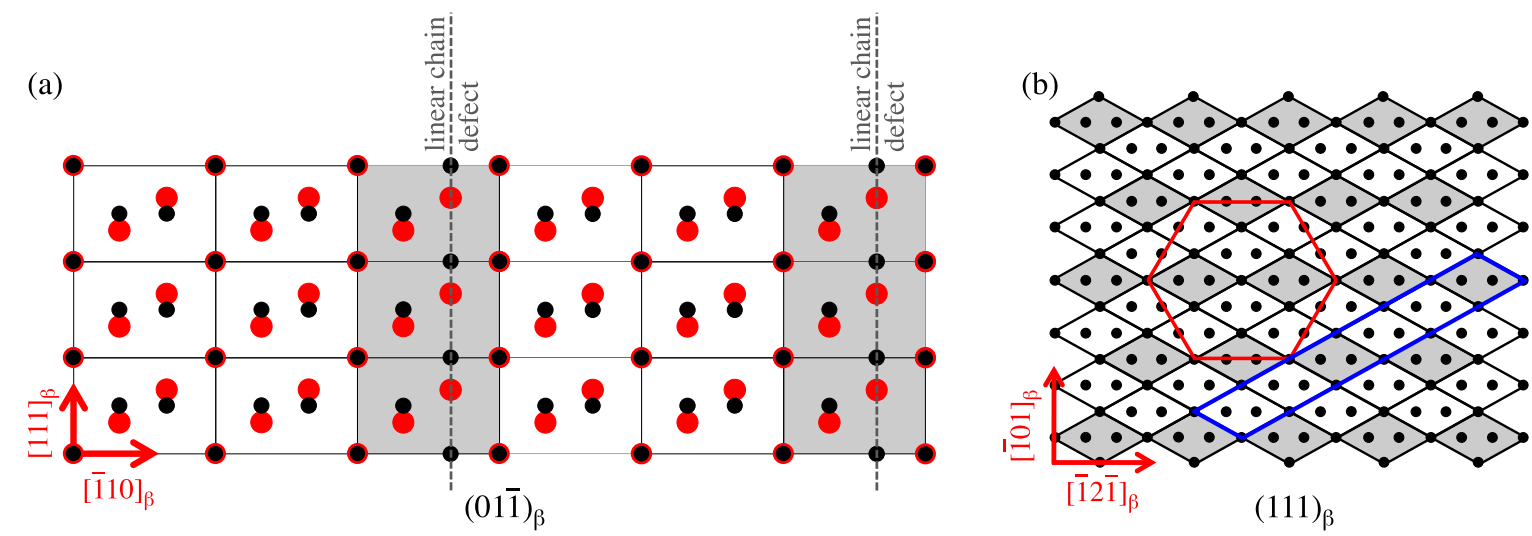

FIG. 4. Illustration of the defective $\omega$ structure found for a $3 \times 3 \times 3$ supercell with 54 atoms employing a projection onto the (a) $(01 \overline{1})_{\beta}$ and (b) $(111)_{\beta}$ plane. Defective $\omega$ unit cells containing a linear-chain defect are displayed in gray color and perfect $\omega$ cells in white. The atoms shown in (a) are emphasized in (b) by the blue colored line. The red lines in (b) emphasize the contour of the $3 \times 3 \times 3$ supercell. [The small relaxations caused by the linear-chain defects are not shown in this figure; see Fig. 2(e) for that purpose.]

an equally dense sampling of the descriptor starting the MD simulations with perfect $\omega$ positions. Corresponding results are shown by the black dots in Fig. 5 and, similarly as for the gray squares, a transition regime around $1000 \mathrm{~K}$ can be identified. Below the transition, the data points are confined to two plateaus corresponding to perfect and defective $\omega$.

For a perfect $\omega$ structure, the descriptor value is

$$
\Delta_{\text {perf }}=\frac{1}{3}\left(0+\frac{1}{6} d_{\mathrm{NN}}+\frac{1}{6} d_{\mathrm{NN}}\right)=\frac{1}{9} d_{\mathrm{NN}}=0.31 \AA .
$$

For a defective $\omega$ structure in the $3 \times 3 \times 3$ supercell with 54 atoms, 6 atoms perform shifts that are larger by $\frac{1}{6} d_{\mathrm{NN}}$ and thus the descriptor amounts to

$$
\Delta_{\text {def }}=\Delta_{\text {perf }}+\frac{6}{54}\left(\frac{1}{6} d_{\mathrm{NN}}\right)=0.37 \AA .
$$

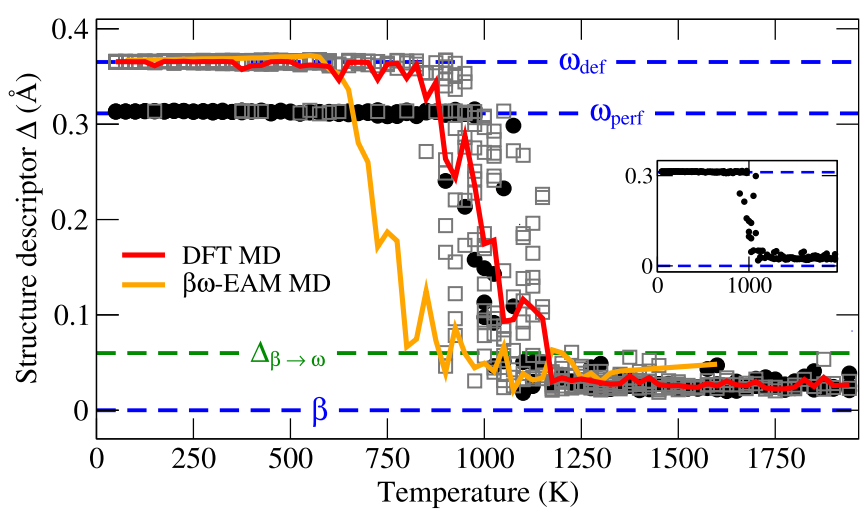

FIG. 5. Temperature dependence of the structure descriptor $\Delta$ [Eq. (1)] for a $3 \times 3 \times 3$ cubic bcc supercell with 54 atoms. Dark gray squares represent DFT MD simulations initialized with $\beta$ positions and black dots with perfect $\omega$ positions. The red line is an average over the gray squares. The orange line shows the corresponding result for the $\beta \omega$-EAM potential. The horizontal dashed green line represents the descriptor value, $\Delta_{\beta \rightarrow \omega}$, used to identify the $\beta$ to $\omega$ transformation. The horizontal dashed blue lines correspond to descriptor values of the $\beta$ structure, the perfect $\omega$ structure $\left(\omega_{\text {perf }}\right)$, and the defective $\omega$ structure $\left(\omega_{\text {def }}\right)$.
When defective $\omega$ structures form, we observe that all atoms undergo a small correlated shift along the symmetry broken $[111]_{\beta}$ direction, opposite to the movement of the defective atoms. This shift is needed to conserve the center of mass of the supercell. To have a well-defined descriptor we subtract this shift from the mean positions prior to applying Eq. (1) to defective $\omega$ structures.

Whether a perfect or defective $\omega$ structure is observed (at low temperatures) depends on the starting positions of the MD simulations. When starting with perfect $\omega$ positions (black dots in Fig. 5) the thermodynamically averaged structure remains in a perfect $\omega$ configuration. When starting with $\beta$ positions (gray squares), a defective $\omega$ structure is observed in most runs and only a small fraction of the runs (about $10 \%)$ shows the perfect $\omega$ structure. To clarify this behavior, we have computed the $T=0 \mathrm{~K}$ DFT energies of the relaxed perfect and defective $\omega$ structures, and the DFT energy barrier between them with nudged elastic band calculations [36,37].

Figure 6 shows that the perfect $\omega$ structure is more stable than the defective one by only $11 \mathrm{meV} /$ atom at $T=0 \mathrm{~K}$. Thus while the perfect $\omega$ structure is the thermodynamic equilibrium state at very low temperatures, the difference in total internal energy per atom is already less than $\frac{1}{2} k_{\mathrm{B}} T$ at ambient

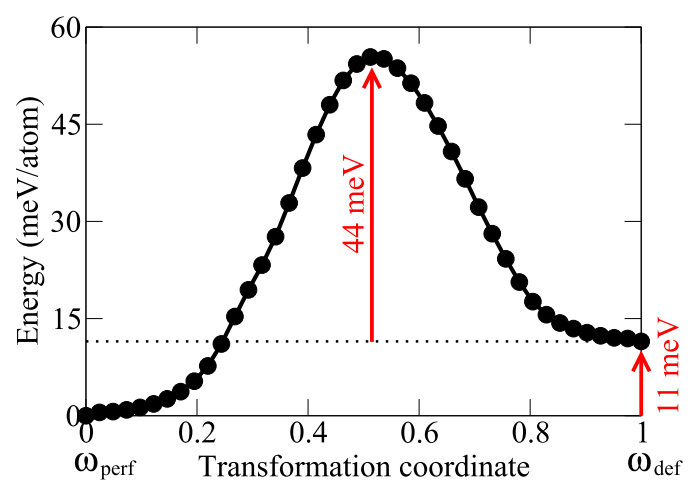

FIG. 6. DFT results for $T=0 \mathrm{~K}$ nudged elastic band calculations between the perfect and defective $\omega$ structures; $\omega_{\text {perf }}$ and $\omega_{\text {def }}$, respectively. 
temperature. We also learn from Fig. 6 that the perfect and the defective $\omega$ structures are separated by an energy barrier of about $50 \mathrm{meV} /$ atom. This is sufficient to inhibit a transition in either direction within the simulation time. Hence, starting with perfect $\omega$ positions the system remains in a perfect $\omega$ structure below the transition to $\beta$ as revealed by the black dots lying on the lower plateau in Fig. 5 ( $\Delta=0.31 \AA$ ).

The reason why the defective $\omega$ structure forms in most cases when starting the MD from $\beta$ positions (gray squares lying on the plateau of $\Delta=0.37 \AA$ ) is related to degeneracy. Coming from the $\beta$ structure the number of symmetrically equivalent configurations is 6 times higher for the defective $\omega$ structure than for the perfect one. The factor of 6 is because the linear-chain defect could run through either of the two middle atoms in the $\omega$ unit cell, which provides a factor 2, and the entire pattern in Fig. 4(a) could be placed in three equivalent positions. The higher number of target configurations makes it statistically more likely for the system to reach the defective $\omega$ structure from the $\beta$ positions. Nevertheless, a few of the simulations do end up in the perfect $\omega$ structure.

Inspection of the data in Fig. 5 above the transformation temperature, i.e., $1000 \mathrm{~K}$ and higher, reveals the stability of the $\beta$ phase. A perfect bcc structure at $T=0 \mathrm{~K}$ would give exactly zero but thermal vibrations in combination with the limited simulation times prevent the descriptor from reaching exactly zero. A time dependent analysis of the structure descriptor at higher temperatures reveals in some simulations of the $3 \times 3 \times 3$ supercell a hopping between symmetrically equivalent $\beta$ structures [cf. Figs. 3(b) and 3(e), $\beta$ and $\beta^{\prime}$ ]. See Ref. [28] for details. However, the hopping is entropically suppressed in larger supercell sizes.

In a recent study, Kadkhodaei et al. [11] likewise investigated bcc Ti in a $3 \times 3 \times 3$ supercell using DFT. They observed deviations of the atomic trajectories from the perfect $\omega$ structure at $1200 \mathrm{~K}$. We interpret their results as corresponding to the defective $\omega$ structure as analyzed above. Kadkhodaei et al. [11] suggested a possible relation with the $\alpha$ (hcp) phase of $\mathrm{Ti}$ that is experimentally known to be stable below $1155 \mathrm{~K}$. We have therefore investigated whether a full $T=$ $0 \mathrm{~K}$ relaxation of the defective $\omega$ structure (using the mean positions from our analysis as a starting point), including atomic as well as cell shape and volume relaxations, would show a tendency towards the $\alpha$ phase. We find, however, that the relaxation is negligible lowering the energy by only $11 \mathrm{meV} /$ atom. We will show in Sec. III C that a geometric connection to the hcp structure can be established for larger supercells.

\section{B. Finite size and finite time effects}

The analysis of the $\beta$ to $\omega$ transformation performed in the previous section was based on the $3 \times 3 \times 3$ supercell with 54 atoms. This supercell has the special property of being commensurate with the $\beta$ and $\omega$ structures (both perfect and defective), and enabled us to determine readily some properties of these phases at the DFT level of accuracy. It is, however, essential to investigate the effect of supercell size on the transformation behavior, which requires MD simulations for larger supercells. Direct DFT MD simulations in larger supercells quickly become prohibitive due to the asymptotically

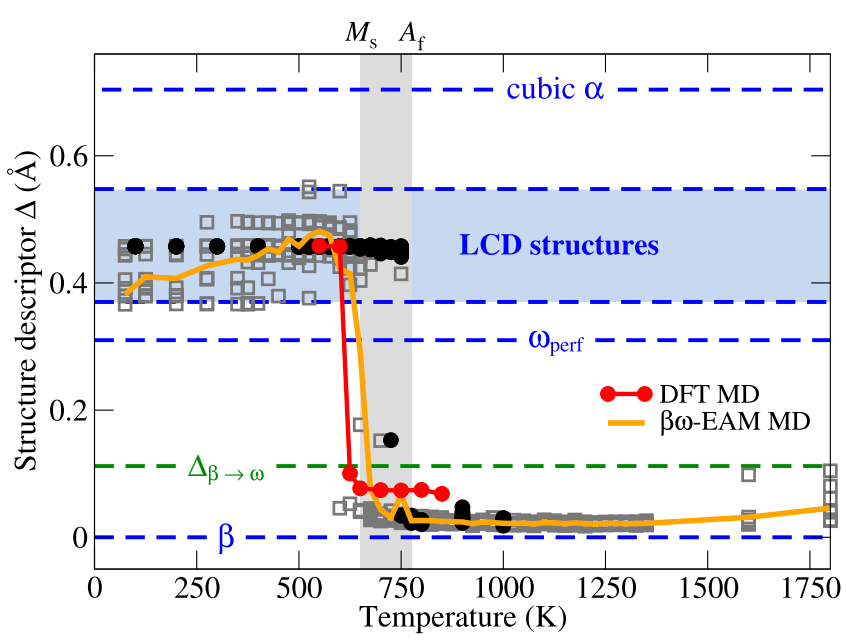

FIG. 7. Temperature dependence of the structure descriptor $\Delta$ [Eq. (1)] for a $6 \times 6 \times 6$ bcc supercell with 432 atoms, starting the MD with $\beta$ positions (dark gray squares) and positions of one particular defective $\omega$ structure (black dots). The correspondence to linear-chain disordered (LCD) structures will be discussed in Sec. IIIC. For comparison purposes similar simulation times are used for both the DFT (5 ps) and $\beta \omega$-EAM (50 ps) calculations. A hysteresis in the transition temperature of about $100 \mathrm{~K}$ is observed.

cubic scaling of CPU time with the number of atoms. We will show in the following that the $\beta \omega$-EAM potential provides a viable alternative for studying much larger supercell sizes and also longer simulation times.

We have first investigated how well the $\beta \omega$-EAM potential reproduces the characteristic features of the DFT MD simulations for the $3 \times 3 \times 3$ supercell. The orange line in Fig. 5 shows the averaged descriptor computed with the $\beta \omega$-EAM potential, using $\beta$ positions as the initial positions, i.e., the orange line can be directly compared with the red line representing DFT. The $\beta$ structure is correctly stabilized at higher temperatures and it correctly transforms to the defective $\omega$ structure when the temperature is reduced. Qualitatively the shape of the $\beta \omega$-EAM curve is close to DFT, although the transition is underestimated by about $200 \mathrm{~K}$.

Using the $\beta \omega$-EAM potential, it is a matter of CPU minutes to calculate the temperature dependence of the structure descriptor for larger supercell sizes. An example for the temperature dependence of the descriptor for a $6 \times 6 \times 6$ supercell with 432 atoms is shown in Fig. 7. The $\beta \omega$-EAM result (orange line) is close in shape and also in the transition temperature to the DFT curve (red line and dots), the latter requiring many orders of magnitude more computational time $(\approx 30000 \mathrm{CPU}$ hours $)$.

For this larger supercell we observe a smaller transformation temperature $(\approx 660 \mathrm{~K})$ and also a more sharply defined transformation regime. Further we see a significant impact of the supercell size below the transformation temperature. Whereas for the smaller $3 \times 3 \times 3$ supercell the descriptor was confined to two plateaus (Fig. 5), the descriptor for the $6 \times 6 \times 6$ supercell shows a range of different values; gray squares in Fig. 7. The reason for the scatter in the descriptor is that larger supercell sizes enable a wealth of new defective $\omega$ structures characterized by different linear-chain defect 
arrangements (discussed in Sec. III C), which can be reached when starting the MD with $\beta$ positions. Only defective $\omega$ structures are observed as indicated by the blue shading (labelled "LCD structures"), whereas the perfect $\omega$ structure no longer appears. This finding can be explained by the much higher number of defective $\omega$ structures.

To investigate the impact of the starting positions, we have selected one specific defective $\omega$ structure using it to initialize the MD simulations. The resultant descriptor is shown by the black dots in Fig. 7. Below the transformation temperature, the system remains located in the initial defective $\omega$ structure as revealed by the single plateau. This indicates that transformations between different defective $\omega$ structures are relatively rare, similarly to the $3 \times 3 \times 3$ supercell.

From Fig. 7, we see that the transformation temperature is about $775 \mathrm{~K}$ for the simulations initiated with a defective $\omega$ structure (black dots). This transformation temperature is about $100 \mathrm{~K}$ higher than the transformation temperature obtained with $\beta$ initial positions which is about $660 \mathrm{~K}$ (orange line). Thus we observe a hysteresis as highlighted by the gray shaded region in Fig. 7. The lower transformation temperature can be associated with a martensite start temperature $M_{\mathrm{s}}$ and the higher with an austenite finish temperature $A_{\mathrm{f}}$. As discussed below, the $\beta \omega$-EAM potential can be used to show that by extending the simulation time the extent of the hysteresis is reduced, such that the hysteresis would vanish if we extrapolated to experimental timescales.

Let us first quantify the impact of the supercell size on $M_{\mathrm{s}}$ and for comparison purposes we use simulation times comparable to our DFT runs $(5, \ldots, 50 \mathrm{ps})$. We define $M_{\mathrm{s}}$ as the temperature where the descriptor value becomes larger than $10 \%$ of the difference between the value for the defective $\omega$ structure and the value for the $\beta$ phase (see green dashed $\Delta_{\beta \rightarrow \omega}$ line in Figs. 5 and 7). The convergence of this temperature with respect to the supercell size and simulation time is shown in Fig. 8. The resulting $M_{\mathrm{S}}$ temperature is shown by the thin orange line with open circles for the $\beta \omega$-EAM potential and by the red line with open squares for DFT. The "error bars" reflect the width of the transition which is significant for small supercells and decreases for larger ones (therefore the error bars are neglected for larger supercells).

We observe a rather strong variation in the $M_{\mathrm{s}}$ temperature of a few hundred degrees Kelvin for the smaller supercell sizes. In particular, the commensurate $3 \times 3 \times 3$ supercell shows up as a peak in the $M_{\mathrm{s}}$ temperature. This peak is somewhat stronger for the DFT curve than for the $\beta \omega$-EAM one, but overall the $\beta \omega$-EAM potential reproduces well the dependence with supercell size.

The quality of the $\beta \omega$-EAM potential in describing the $\beta$ to $\omega$ transformation is reflected by comparison to results obtained with a modified EAM (MEAM) potential available from literature [18]. The potential of Ref. [18] was obtained by fitting to a wide range of properties, e.g., energies, elastic constants and forces for a variety of Ti phases and has been in particular shown to reproduce accurately the experimental temperature-pressure phase diagram of Ti, including the $\alpha, \beta$, and $\omega$ phases. The $M_{\mathrm{S}}$ temperature from $\beta$ to $\omega$ calculated with this potential is shown in Fig. 8 by the gray line. While the qualitative dependence with supercell size is reasonably close to the $\beta \omega$-EAM and DFT curves, the absolute $M_{\mathrm{s}}$

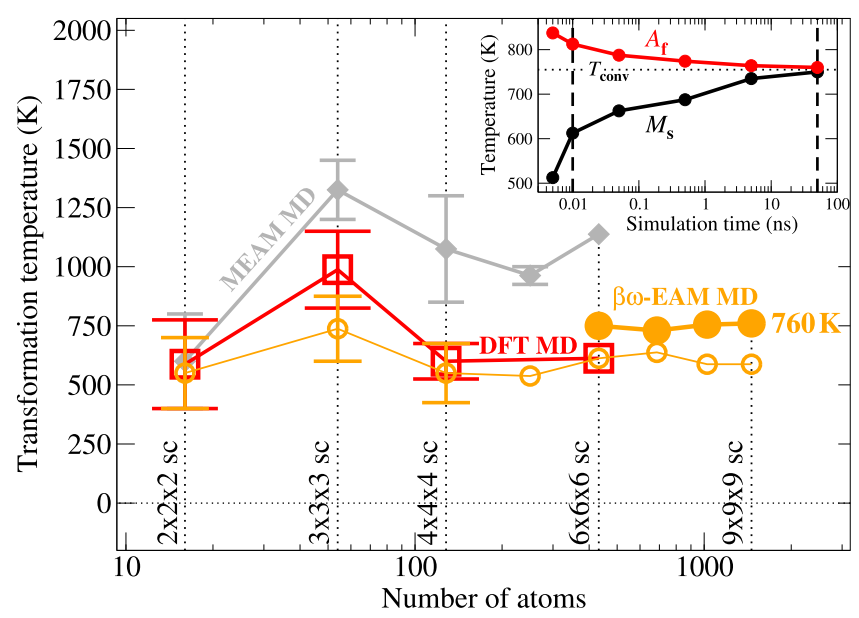

FIG. 8. Supercell size dependence of the $\beta$ to $\omega$ transformation temperature $\left(M_{\mathrm{s}}\right)$ employing DFT (red), our optimized $\beta \omega$-EAM potential (orange; both thin and thick lines), and a MEAM potential from literature (gray) [18]. The thin orange line with open circles and the gray line correspond to DFT-accessible simulation times $(5, \ldots, 50 \mathrm{ps})$ and the thick orange line with dots corresponds to converged simulation times of $50 \mathrm{~ns}$. For the $6 \times 6 \times 6$ supercell (432 atoms), the $\beta \omega$-EAM transformation temperature is $613 \pm 13 \mathrm{~K}$ after $10 \mathrm{ps}$ (matching well the corresponding DFT value) and $750 \pm$ $10 \mathrm{~K}$ after $50 \mathrm{~ns}$. The error bars indicate the transition region (cf. Fig. 5) which decreases with increasing system size. The inset shows the convergence of $M_{\mathrm{s}}$ and $A_{\mathrm{f}}$ in the $6 \times 6 \times 6$ supercell with the two vertical dashed lines indicating DFT-accessible (left) and converged simulation times (right).

temperatures are overestimated for the larger cell sizes. Thus, for the particular application to the $\beta$ to $\omega$ transformation, the present $\beta \omega$-EAM potential is better suited.

Having analyzed the $M_{\mathrm{s}}$ dependence on the system size we now turn to the question of how $M_{\mathrm{s}}$ and also $A_{\mathrm{f}}$ depend on the simulation time, i.e., how the hysteresis changes with longer simulation times. Direct DFT MD simulations are computationally prohibitive for that purpose, specifically for the larger supercells, and the availability of the accurate $\beta \omega$ EAM potential is therefore critical.

Results for the $6 \times 6 \times 6$ supercell are shown in the inset of Fig. 8. With increasing simulation time the hysteresis decreases, and the two temperatures $M_{\mathrm{s}}$ and $A_{\mathrm{f}}$ converge towards a single temperature, $T_{\text {conv }} \approx 760 \mathrm{~K}$. After a simulation time of $50 \mathrm{~ns}, M_{\mathrm{s}}$ and $A_{\mathrm{f}}$ are converged to within $10 \mathrm{~K}$. The convergence behavior is asymmetric: $A_{\mathrm{f}}$ converges comparably quickly to within $1 \mathrm{~K}$ already within the DFT simulation timescale (ps), while the convergence for $M_{\mathrm{S}}$ is significantly slower, requiring simulation times on the ns scale to converge as well as $A_{\mathrm{f}}$. It is therefore important to consider carefully the simulation-time dependence when analyzing the transformation behavior. Fully time-converged results for the transformation temperature are shown in Fig. 8 by the orange dots, revealing that the $6 \times 6 \times 6$ supercell already gives a transformation temperature that is converged well with supercell size $(750 \mathrm{~K}$ versus $760 \mathrm{~K}$ for the $9 \times 9 \times 9$ supercell). 
(a)

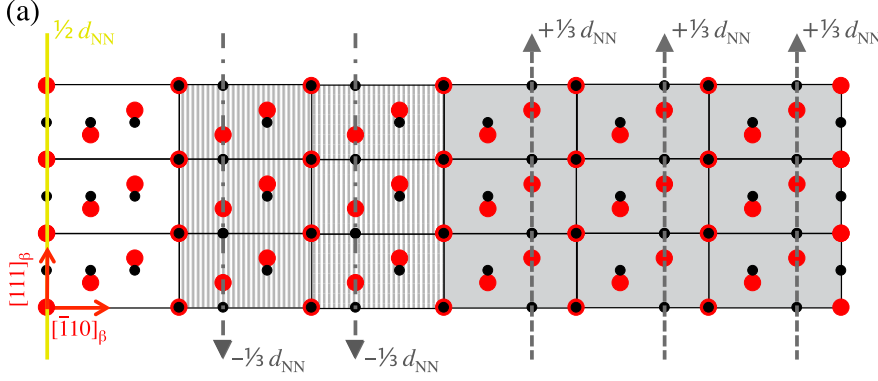

$(01 \overline{1})_{\beta}$

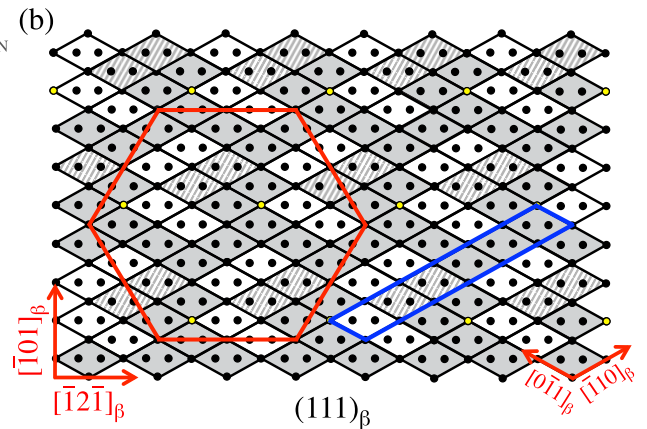

FIG. 9. Example of a defective $\omega$ structure in a $6 \times 6 \times 6$ supercell with 432 atoms in a (a) $(01 \overline{1})_{\beta}$ and (b) (111) $\beta$ projection. The cells shown in (a) are highlighted in (b) by the blue contour. The red lines in (b) emphasize the contour of the $6 \times 6 \times 6$ supercell. Three possible $[111]_{\beta}$ linear-chain defects in a $\omega$ unit cell are present for this $\omega$ structure: (1) one of the middle atoms shifted downward by $1 / 3 d_{\mathrm{NN}}$ with $d_{\mathrm{NN}}$ the nearest neighbor distance in $\beta$ (gray striped cells), (2) the other middle atom shifted upward by $1 / 3 d_{\mathrm{NN}}$ (gray shaded cells), and (3) corner atom shifted by $1 / 2 d_{\mathrm{NN}}$ [yellow line in (a) and yellow dots in (b)].

\section{Linear-chain disordered (LCD) structures}

To understand the geometric nature of the observed dependence of the transformation temperature on the supercell size, we have analyzed the mean MD positions from the various supercells. A main outcome of this analysis is that the finite sizes of the smaller supercells and the accompanying periodic boundary conditions impose geometric restrictions on the $\omega$ phase. These restrictions are gradually lifted when going to larger system sizes. One aspect is related to the commensurability of the $\beta$ and $\omega$ structures. For $3 n \times 3 n \times 3 n$ supercells where $n$ is an integer, the $\beta$ and $\omega$ structures are perfectly commensurate. For noncommensurable supercells (e.g., $4 \times 4 \times 4$ or $5 \times 5 \times 5$ ) geometric incompatibilities are compensated by interface defects between the $\omega$ unit cells and a shear strain component along the $[111]_{\beta}$ direction. The $\omega$ phase is destabilized and the transformation temperature lowered. Further details are given in Ref. [28]. The contribution of interface defects becomes however readily less important with increasing supercell size. A much more important aspect is related to the formation of $[111]_{\beta}$ linear-chain defects in the $\omega$ phase, for which the number of different types and spacial arrangements increases. This aspect will lead us to the definition of a new set of structures, the linear-chain disordered (LCD) structures.

For the supercell size comprising $6 \times 6 \times 6$ cubic bcc unit cells with 432 atoms, one may have expected a similar transformation behavior as for the $3 \times 3 \times 3$ supercell. The $6 \times$ $6 \times 6$ supercell is commensurate, containing exactly $432 / 3=$ $144 \omega$ unit cells, thus in principle not requiring any geometric defects. Indeed, as expected there are no interface defects and no shear strain present. However, there is a significant number of $[111]_{\beta}$ linear-chain defects present and, importantly, these linear-chain defects exhibit new characteristics as compared to the $3 \times 3 \times 3$ supercell: (i) different types of linear-chain defects can occur concurrently in the same supercell and (ii) there are significantly more arrangements of the linear-chain defects in the symmetry-broken $(111)_{\beta}$ plane.

Figure 9 shows an example of the three types of $[111]_{\beta}$ linear-chain defects feasible for the $6 \times 6 \times 6$ supercell and a possible arrangement in the $(111)_{\beta}$ plane. Two types of these linear-chain defects have been observed already for the $3 \times 3 \times 3$ supercell, however not simultaneously in the same supercell. They correspond to the two inner atoms of the $\omega$ unit cell shifted in opposite $[111]_{\beta}$ directions (gray stripes and shading in Fig. 9). These linear-chain defects can be assigned an up or down direction as emphasized in Fig. 9(a) by the arrows. The third, new linear-chain defect corresponds to a shift of a corner atom of the $\omega$ unit cell by $\mathrm{d}_{\mathrm{NN}} / 2$ along the $[111]_{\beta}$ direction (yellow lines and dots in Fig. 9). The linear-chain defects are arranged in a specific pattern in the $(111)_{\beta}$ plane that preserves translational symmetry of the supercell. There are many ways of placing this pattern inside the $6 \times 6 \times 6$ supercell. Moreover, the pattern shown in Fig. 9(b) is just one particular example among many. In other $6 \times 6 \times 6$ supercell simulations (different temperatures or initial configurations), we observe different, symmetrically inequivalent patterns.

The difference in the linear-chain defects and their arrangements between the $3 \times 3 \times 3$ and $6 \times 6 \times 6$ supercells is associated with their different transformation temperatures as observed in our MD simulations (Fig. 8). The fact that the transformation temperature for the $6 \times 6 \times 6$ supercell is lower suggests that the defective $\omega$ structure in this supercell is destabilized energetically as compared to the defective $\omega$ structure in the $3 \times 3 \times 3$ supercell. Put differently, the artificial long-range order imposed by the periodic boundary conditions in the $3 \times 3 \times 3$ supercell stabilizes the defective $\omega$ structure and thus increases its transformation temperature.

For supercells larger than $6 \times 6 \times 6$, the number of types of $[111]_{\beta}$ linear-chain defects remains at three, but the number of the different arrangements of the defects rapidly increases. A few representative examples are shown in Fig. 10 for a $12 \times 12 \times 12$ supercell. There seems to be no obvious correlation between the linear-chain defects over longer distances within the supercell. The linear-chain defects rather appear in randomly disordered arrangements and we therefore define the set of such structures as linear-chain disordered (LCD) structures. When an LCD structure is formed, atoms move randomly between the original triangular and hexagonal planes characterizing $\beta$ and $\omega$ (cf. Figs. 1(b) and 1(c); more details are given in Ref. [28]). The gross effect of the randomness in the LCD structures on the overall energetics and dynamics becomes smaller beyond the $6 \times 6 \times 6$ supercell as evidenced by the small changes in the transformation 


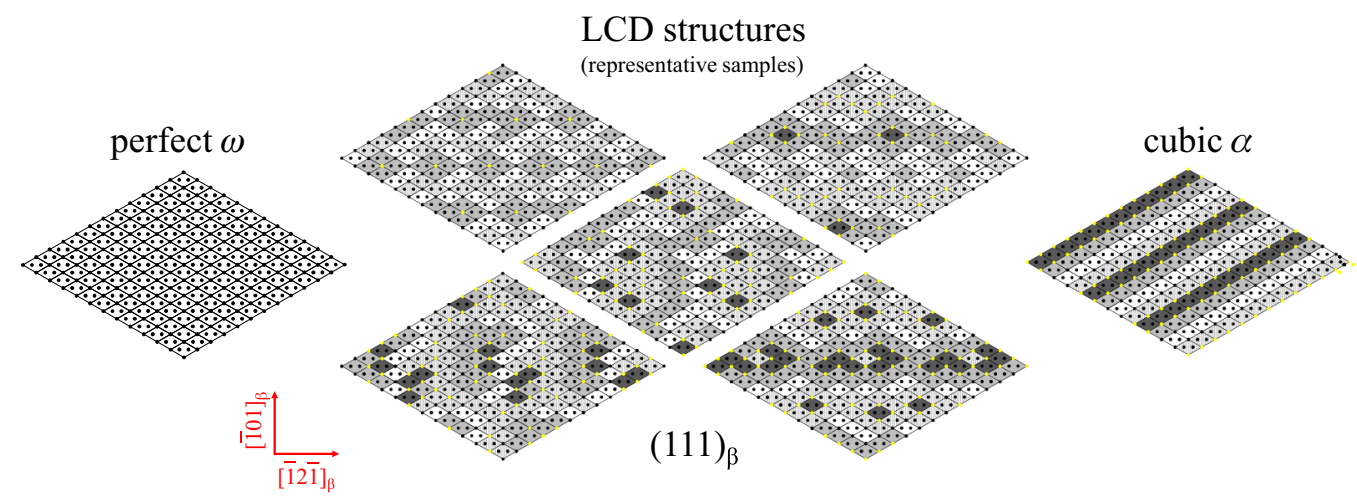

FIG. 10. Representative examples of LCD structures obtained from MD simulations in a $12 \times 12 \times 12$ supercell at $T=500 \mathrm{~K}$. The three types of possible linear-chain defects are the same as in the $6 \times 6 \times 6$ supercell. In contrast to the $6 \times 6 \times 6$ supercell the up and down linear-chain defects can occur in the same $\omega$ unit cell (dark gray shaded cells). The perfect $\omega$ structure (left) contains no linear-chain defects and the cubically constrained $\alpha$ structure (right) is made of long-ranged ordered defects. Note that the statistical weight of the LCD structures is large compared to the perfect $\omega$ and cubic $\alpha$ structure and that the latter two thus do not occur in the simulations.

temperature (Fig. 8). A $6 \times 6 \times 6$ supercell is therefore a reasonably well converged supercell size to study the properties of LCD structures.

Based on the concept of the newly introduced LCD structures we can draw a connection to the $\alpha$ (hcp) phase. For a specific arrangement of the linear-chain defects with strong long-range order as shown in Fig. 10 to the right, we obtain a cubically constrained $\alpha$ structure, i.e., by applying an additional strain deformation we would reach the actual hexagonal $\alpha$ phase. Such a transformation is related to the Silcock pathway from $\alpha$ to $\omega$ as studied in Ref. [38]. Note that the cubic $\alpha$ structure is only commensurable with the $\beta$ structure for a $12 n \times 12 n \times 12 n$ supercell $(n=$ integer $)$. The structure descriptor of the cubic $\alpha$ structure corresponds to $\Delta_{\alpha}=0.704 \AA$ and gives an upper bound to structure descriptors for the LCD structures. The lower bound is given by the perfect $\omega$ structure (cf. Fig. 7). We stress, however, that neither the perfect $\omega$ nor the cubic $\alpha$ structure are found in our simulations. The statistical weight of the LCD structures strongly dominates over these special structures.

\section{CONCLUSION}

We have demonstrated an efficient atomistic approach to study dynamically unstable systems with DFT accuracy over the full temperature range, i.e., including the high- and low-temperature stable phases as well as the transformation regime. At the core of this approach is an interatomic potential that is fitted to the most relevant part of the phase space, i.e., a set of DFT MD energies for the high- and lowtemperature phases. Transferability to other parts of the phase space (e.g., other phases) is not sought, which is in contrast to conventional interatomic potential fitting. With our fitting strategy, the potential provides better accuracy for the target quantities than potentials that are fitted to be more versatile. In particular, it provides accurate transformation temperatures and atomistic features of the transformation.

The parametrization employed here for the interatomic potential has been the embedded atom method ( $\beta \omega$-EAM potential) with the fitting to DFT MD energies performed using the MEAMFIT2 code [26,27], which is freely available for academic use. Other parametrizations could be employed as well, for example modified EAM which is also available in MEAMFIT2 (in a reference-free implementation). Other possibilities are Gaussian approximation potentials [39] or machine-learning potentials [40]. Such more advanced parametrizations offer a higher degree of flexibility and thus the possibility for further accuracy improvement, albeit at an increase in fitting complexity and computational costs. Since any of these potentials are orders of magnitude faster to compute than DFT, they enable one to study the impact of finite size and finite time effects on the transformation behavior, both of which turn out to be critical.

We have applied our approach to the prototypical dynamically unstable $\beta$ (bcc) phase in Ti and studied its transformation to the low-temperature $\omega$ phase. Our analysis has led us to the introduction of a new set of structures, the linear-chain disordered (LCD) structures. These structures are suppressed by the periodic boundary conditions in the small supercells that are accessible with DFT calculations. At least a $6 \times 6 \times 6$ supercell is required to provide enough freedom to the system to produce LCD structures. The LCD structures can be derived from the perfect $\omega$ structure by considering three types of linear-chain defects along the $[111]_{\beta}$ direction. These linearchain defects correspond to three different shifts of atoms in the primitive $\omega$ unit cell. These are shifts of $\pm 1 / 3$ or $1 / 2$ of the nearest neighbor distance in $\beta$, precisely the three possible shifts that preserve the surrounding $(111)_{\beta}$ planes, corresponding to the three variants of $\omega$ in this direction. The linear-chain defects appear randomly disordered when viewed in the $(111)_{\beta}$ plane. With the concept of the linear-chain defects also a cubically constrained $\alpha$ (hcp) phase can be produced theoretically. Neither the perfect $\omega$ nor the cubic $\alpha$ structure are observed in the simulations due to the statistical dominance of the LCD structures.

Based on the present results it is not possible to say whether the linear-chain defects will persist for experimental timescales and macroscopic system sizes. However, for all our simulation times we observe that the linear-chain defects are quenched in. 
Another interesting open question relates to the nucleation process of the linear-chain defects along the $[111]_{\beta}$ direction. Our results indicate a correlated movement during the creation of a linear-chain defect. For macroscopically large system sizes such a correlated movement will be entropically suppressed. We expect that a localized nucleation process will take over, possibly enhanced by vacancies. It is conceivable that a vacancy-assisted migration of the linear-chain defects may be related to the anomalous diffusion behavior observed in $\mathrm{Ti}$ [41].

We expect that the results presented here for the prototypical $\beta$ Ti phase apply to the $\omega$ transition in real alloys of Ti and $\mathrm{Zr}$, in which the $\omega$ phase is known to form and to contribute to mechanical properties depending on alloy composition and heat treatment $[42,43]$. In general terms, such defect formation may occur in other kinds of dynamically unstable bec based systems. Preliminary results support this statement [44].

\section{ACKNOWLEDGMENTS}

Funding from the European Research Council (ERC) under the EU's Horizon 2020 Research and Innovation Programme (Grant No. 639211) is gratefully acknowledged. A.I.D. and M.W.F. would like to thank the EPSRC Program Grant (Grant No. EP/K008749/1) Material Systems for Extreme Environments (XMat) for financial support. M.W.F. also acknowledges the support of an Alexander von Humboldt Award and A.I.D. the support of the STFC Hartree Centre's Innovation: Return on Research programme, funded by the UK Department for Business, Energy \& Industrial Strategy.
[1] P. J. Craievich, M. Weinert, J. M. Sanchez, and R. E. Watson, Phys. Rev. Lett. 72, 3076 (1994).

[2] K. Einarsdotter, B. Sadigh, G. Grimvall, and V. Ozolins, Phys. Rev. Lett. 79, 2073 (1997).

[3] V. Ozolins, Phys. Rev. Lett. 102, 065702 (2009).

[4] G. Grimvall, B. Magyari-Köpe, V. Ozolins, and K. A. Persson, Rev. Mod. Phys. 84, 945 (2012).

[5] P. Hohenberg and W. Kohn, Phys. Rev. 136, B864 (1964).

[6] P. Souvatzis, O. Eriksson, M. I. Katsnelson, and S. P. Rudin, Phys. Rev. Lett. 100, 095901 (2008).

[7] P. Souvatzis, O. Eriksson, M. I. Katsnelson, and S. P. Rudin, Comput. Mater. Sci. 44, 888 (2009).

[8] O. Hellman, P. Steneteg, I. A. Abrikosov, and S. I. Simak, Phys. Rev. B 87, 104111 (2013).

[9] A. van de Walle, Q. Hong, S. Kadkhodaei, and R. Sun, Nat. Commun. 6, 7559 (2015).

[10] J. C. Thomas and A. Van der Ven, Phys. Rev. B 88, 214111 (2013).

[11] S. Kadkhodaei, Q.-J. Hong, and A. van de Walle, Phys. Rev. B 95, 064101 (2017).

[12] S. Kadkhodaei and A. van de Walle, Acta Mater. 147, 296 (2018).

[13] A. I. Duff, T. Davey, D. Korbmacher, A. Glensk, B. Grabowski, J. Neugebauer, and M. W. Finnis, Phys. Rev. B 91, 214311 (2015).

[14] L.-F. Zhu, B. Grabowski, and J. Neugebauer, Phys. Rev. B 96, 224202 (2017).

[15] L.-F. Huang, B. Grabowski, E. McEniry, D. R. Trinkle, and J. Neugebauer, Phys. Status Solidi B 252, 1907 (2015).

[16] D. A. Young, Phase Diagrams of the Elements (University of California Press, Berkeley, 1991).

[17] J. Zhang, Y. Zhao, R. S. Hixson, G. T. Gray III, L. Wang, W. Utsumi, S. Hiroyuki, and H. Takanori, J. Phys. Chem. Solids 69, 2559 (2008).

[18] R. G. Hennig, T. J. Lenosky, D. R. Trinkle, S. P. Rudin, and J. W. Wilkins, Phys. Rev. B 78, 054121 (2008).

[19] Z.-G. Mei, S.-L. Shang, Y. Wang, and Z.-K. Liu, Phys. Rev. B 80, 104116 (2009).
[20] C.-E. Hu, Z.-Y. Zeng, L. Zhang, X.-R. Chen, L.-C. Cai, and D. Alfè, J. Appl. Phys. 107, 093509 (2010).

[21] L.-F. Huang, B. Grabowski, J. Zhang, M.-J. Lai, C. C. Tasan, S. Sandlöbes, D. Raabe, and J. Neugebauer, Acta Mater. 113, 311 (2016).

[22] T. Li, D. Kent, G. Sha, L. T. Stephenson, A. V. Ceguerra, S. P. Ringer, M. S. Dargusch, and J. M. Cairney, Acta Mater. 106, 353 (2016).

[23] D. J. Lin, J. H. Chern Lin, and C. P. Ju, Mater. Chem. Phys. 76, 191 (2002).

[24] M. Friák, W. A. Counts, D. Ma, B. Sander, D. Holec, D. Raabe, and J. Neugebauer, Materials 5, 1853 (2012).

[25] H. E. Cook, Acta Metall. 21, 1445 (1973).

[26] A. I. Duff, M. W. Finnis, P. Maugis, B. J. Thijsse, and M. H. F. Sluiter, Comp. Phys. Commun. 196, 439 (2015).

[27] MEAMFIT2 is an interatomic potential optimization package and has been obtained from STFC's Daresbury Laboratory via the website https://www.scd.stfc.ac.uk/Pages/MEAMfit-v2.aspx.

[28] See Supplemental Material at http://link.aps.org/supplemental/ 10.1103/PhysRevB.100.104110 for more information regarding the parametrization of the $\beta \omega$-EAM potential, the hopping between symmetrically equivalent $\beta$ structures, the defective triangular and hexagonal planes, and the behavior of noncommensurable system sizes. Supplemental Material contains Refs. [26], [27], [45].

[29] G. Kresse and J. Furthmüller, Phys. Rev. B 54, 11169 (1996).

[30] G. Kresse and J. Furthmüller, Comput. Mater. Sci. 6, 15 (1996).

[31] P. E. Blöchl, Phys. Rev. B 50, 17953 (1994).

[32] J. P. Perdew, K. Burke, and M. Ernzerhof, Phys. Rev. Lett. 77, 3865 (1996).

[33] G. Kresse and D. Joubert, Phys. Rev. B 59, 1758 (1999).

[34] S. Plimpton, J. Comput. Phys. 117, 1 (1995).

[35] D. de Fontaine, Acta Metall. 18, 275 (1970).

[36] G. Henkelman, B. P. Uberuaga, and H. Jonsson, J. Chem. Phys. 113, 9901 (2000).

[37] G. Henkelman and H. Jonsson, J. Chem. Phys. 113, 9978 (2000).

[38] J. M. Silcock, Acta Metall. 6, 481 (1958). 
[39] A. P. Bartók, M. C. Payne, R. Kondor, and G. Csányi, Phys. Rev. Lett. 104, 136403 (2010).

[40] E. V. Podryabinkin and A. V. Shapeev, Comput. Mater. Sci. 140, 171 (2017).

[41] U. Köhler and C. Herzig, Phys. Status Solidi B 144, 243 (1987).
[42] W.-F. Ho, J. Med. Eng. 28, 47 (2008).

[43] S. K. Sikka, Y. K. Vohra, and R. Chidambaram, Prog. Mater. Sci. 27, 245 (1982).

[44] S. Bigdeli, Ph.D. thesis, KTH Royal Institue of Technology, 2017.

[45] M. S. Daw and M. I. Baskes, Phys. Rev. B 29, 6443 (1984). 